\title{
Temporal patch occupancy dynamics of the Siberian flying squirrel in a boreal forest landscape
}

\author{
Eija Hurme, Mikko Mönkkönen, Pasi Reunanen, Ari Nikula and Vesa Nivala \\ E. Hurme (Eija.Hurme@oulu.fi) and P. Reunanen, Dept of Biology, P.O. Box 3000, FI-90014 Univ. of Oulu, Finland. - M. Mönkkönen, \\ Dept of Biological and Environmental Science, P.O. Box 35, FI-40014 Univ. of Jyväskylä, Finland. - A. Nikula and V. Nivala, Finnish Forest \\ Research Inst., Rovaniemi Research Station, P.O. Box 16, FI-96301 Rovaniemi, Finland.
}

\begin{abstract}
Ability to predict species distribution in a landscape is of crucial importance for natural resource management and species conservation. Therefore, the understanding of species habitat requirements and spatio-temporal dynamics in occurrence is needed. We examined patch occupancy patterns of the Siberian flying squirrel Pteromys volans in northern Finland across a seven year study period. Forest patches dominated by mature spruce (Picea abies) in a study area $\left(375 \mathrm{~km}^{2}\right)$ were surveyed to monitor the presence or absence of the flying squirrel. The patch occupancy pattern was dynamic: about half of the habitat patches were occupied at least once during the study period and more patches were colonised than were abandoned. Patches that were continuously occupied (i.e. occupied during all sample periods) were typically of high quality (based on habitat and landscape characteristics), continuously unoccupied patches were usually of low quality, and intermediate quality patches were occupied intermittently. The variables explaining patch occupancy were similar each year, and a statistical model based on data from the year 2000 also predicted occupancy in 2004 with similar accuracy. However, data from a single survey were inadequate for identifying patches used intermittently by flying squirrels. Despite inconsistent occupancy, these patches may be important for the local persistence of flying squirrels. The dynamic occupancy pattern may thus affect estimates of suitable habitat area and identification of functional patch networks for landscape planning. These results emphasise the need for follow-up studies to better understand population patterns and processes in time.
\end{abstract}

The ability to correctly predict species distribution in a landscape is of crucial importance for natural resource management and species conservation. Therefore, understanding species habitat requirements is necessary. Nichebased distribution models relate observations of species to attributes of the environment and result in a set of predictors directly or indirectly reflecting the occurrence of the species (Guisan and Zimmermann 2000, Scott et al. 2002). However, distribution models are often static since they assume that a response by a species to its habitat is constant over time (Randin et al. 2006). In reality, a species distribution changes over time according to, for example, population density. It is therefore important to know if the same attributes of the environment can be used to predict the species distribution when population density is both high and low, and if data from a single year survey are adequate to describe important environmental factors that determine the species distribution (van Apeldoorn et al. 1994, Verbeylen et al. 2003, Guisan and Thuiller 2005).

Species abundance data are usually preferred for conservation purposes because it confers more information than mere presence and absence (Tosh et al. 2004). However, species of special conservation interest are often scarce and difficult to detect. Thus, presence-absence surveys may be the only feasible option for monitoring species distribution. The occupancy rate of habitat patches within a certain area may reflect the species abundance, and the positive associations between occupancy rate and abundance seem to be common (Gaston et al. 2000). Some recent studies suggest that presence-absence data can be as powerful or more powerful than abundance data in detecting population changes, especially when financial resources are limited (Nielsen et al. 2005, Pollock 2006, Joseph et al. 2006). Surveying species presence-absence may thus be a very effective approach for conservation biology and landscape management planning given the difficulty and higher costs involved in collecting the abundance data.

If species occupancy rate and abundance are positively associated, one may expect that some low quality habitat patches will be occupied only in years of high abundance and correspondingly, only high quality habitat patches will be occupied in years of low abundance. Knowledge of longterm species distribution may thus be required to evaluate the relative importance of individual patches, which may not be evident based on a single-year survey. Changes in patch occupancy may reflect patterns and processes related 
to demography, predators, food, diseases, weather, or changes in habitat availability and landscape configuration. The crowding effect (sensu Lovejoy et al. 1986), which denotes an influx of individuals to refuges of remaining habitat patches due to a sudden habitat loss such as clear cuts in forests, may also be involved. Therefore, colonisation of low quality habitats can be due to both an increase in population density and habitat loss.

We focused on the Siberian flying squirrel Pteromys volans, which is an arboreal rodent inhabiting mature spruce (Picea abies)-dominated mixed forests in Eurasian taiga. The flying squirrel is categorized as a vulnerable species in Finland and its habitats are protected due to an apparent population decline during recent decades (Rassi et al. 2001). Some of the flying squirrel's ecological characteristics such as preferred habitat and home range sizes (Mönkkönen et al. 1997, Hanski et al. 2000, Reunanen et al. 2000), dispersal ability (Selonen and Hanski 2004, 2006) and habitat responses at the landscape scale (Reunanen et al. 2000, 2002b), are relatively well studied in Finland. However, long-term studies that focus on dynamics in distribution or abundance are lacking.

The flying squirrel is nocturnal and moves through the forest canopy principally by gliding from tree to tree. Sightings of individuals are rare, so patch occupancy determination is typically based on observation of faecal pellet accumulations under large spruce and aspen Populus tremula trees (Reunanen et al. 2002a). To get an overview of the possible variation in patch occupancy within a relatively large landscape, we assessed patch occupancy every second year across a seven year period.

We examined if the species occupancy was consistently related to habitat patch variables and landscape structure among years. A large variation in occupancy status and in variables that explain the occupancy would render singleyear data inapplicable. We also tested if variables that were related to occupancy status in one year can reliably be used to predict occupancy in another year. We further address whether temporal changes in patch occupancy were associated with landscape structure and change. Finally, we discuss the importance of follow-up studies and the dynamics of occupancy in terms of habitat patch network and landscape management planning.

\section{Material and methods}

\section{Forest data and habitat classification}

Our study area is located in northern Finland $\left(65^{\circ} 30^{\prime} \mathrm{N}\right.$, $28^{\circ} 15^{\prime} \mathrm{E}$ ) and is $374.6 \mathrm{~km}^{2}$ in size (see also Reunanen et al. 2004, Hurme et al. 2007). The landscape belongs to the northern boreal vegetation zone (Ahti et al. 1968), and is characterised by forested ridges with open fens, pine bogs and small lakes in the lowlands. Fields and settlement are scarce, and some forest roads exist. Approximately $1 \%$ of the total forest area is clear-cut annually (based on a $100 \mathrm{yr}$ rotation). The topography varies between 180 and $380 \mathrm{~m}$ a.s.l. Coniferous forests cover ca $70 \%$ of the study area and are principally dominated by Scots pine Pinus sylvestris and Norway spruce, with interspersed deciduous trees (birch
Betula sp., aspen and alder Alnus incana). The majority of forests are $<60 \mathrm{yr}$ old, and ca $25 \%$ are $>100 \mathrm{yr}$ old.

A land cover map of the study area was produced for GIS analysis based on multi-source national forest inventory (MS-NFI) data (see also Hurme et al. 2007). The MS-NFI data are primarily derived from Landsat TM 5 satellite images, where the land and forests are characterised in $25-\mathrm{m}$ pixels and estimates for forest are further verified based on ground reference plots (Tomppo 1993). Since the original satellite images were recorded in 1993, MS-NFI data was updated by incorporating information on forest cutting between 1993 and 2004 from forest planning data of Metsähallitus (a federal enterprise governing state owned land and waters). Then, from the perspective of the flying squirrel's habitat needs, we classified the land cover types into two ecological categories consisting of potential habitat $(\mathrm{Ph})$ and non-habitat matrix (Matrix).

Classification of flying squirrel habitat (described below) was consistent with previous studies (after Reunanen et al. 2002b, 2004, Hurme et al. 2007). The home ranges of flying squirrels, averaging 60 ha for males and 8 ha for females (Hanski et al. 2000), consist of several core areas rich in deciduous trees (Hanski 1998). Therefore, to identify core habitats that reflect preferred forest characteristics by the flying squirrel, we classified pixels that consisted of mature spruce-dominated forest with a mixture of deciduous trees as core-pixels. Buffers (50-m radius, which can be crossed by gliding; see further details from Hurme et al. 2007) were created around each of these "core-habitat" pixels, and all core-habitat with buffers that adjoined or overlapped were united to form a "patch" of potential habitat. Thus, a habitat patch likely reflects qualitatively suitable forest from the perspective of the flying squirrel. However, in our landscape analysis we considered only habitat patches $>3.5$ ha (area of core pixels $\geq 1 \mathrm{ha}$ ) since smaller forests are too small to support a female breeding territory (Hanski et al. 2000, Selonen et al. 2001). The remaining landscape was assigned as matrix for the flying squirrel, covering all other forests, clear cuts, saplings, bogs, lakes and fields. In an earlier study, Hurme et al. (2007) showed that patch occupancy was primarily a function of patch size, patch quality and inter-patch distances, and that matrix quality was of less importance in our study area. Therefore, we did not consider matrix properties in more detail here.

The habitat patches were surveyed for presence and absence of the flying squirrel every second year. Patch occupancy was determined based on presence of faecal pellets (Reunanen et al. 2002a). Absence of the species, i.e. a lack of pellets, was confirmed by surveying the whole habitat patch thoroughly without time limits. The two-year interval in our surveys was to ensure the decomposition of pellets and to minimize the probability that the same pellets (in dry sites) would have indicated the species presence in past years. Due to the relatively easy detectability of pellets, we assumed that the risk of assigning an occupied habitat patch as unoccupied was negligible. Every habitat patch was visited once in June 2000, and the survey was repeated in June 2002 as well as June 2004. Only the habitat patches with a change in occupancy status during 2000-2004 were surveyed for a fourth time in June 2006. The same experienced individuals carried out the field surveys. 


\section{Landscape metrics}

We described the characteristics of habitat patches, matrix and connections to neighbouring patches using several variables. The quality of each habitat patch was defined based on size (Patch size; ha), the combined area of corehabitat pixels (Core-area; ha), the proportion of core pixels relative to the total patch area (Core-pro), and the growing stock volumes $\left(\mathrm{m}^{3} \mathrm{ha}^{-1}\right)$ of spruce (Spruce) and deciduous trees (Deciduous) within a patch. The quality of the landscape surrounding each patch was defined as the proportion of potential habitat $(\mathrm{Ph} 500)$ and non-habitat (Matrix500) within a 500-m buffer. This buffer radius was selected because it is roughly equivalent to the nocturnal activity area of an individual flying squirrel (Hanski et al. 2000, Selonen and Hanski 2006). Furthermore, there is minimal overlap among buffers of this size, which helped to maintain independence among metrics describing individual patches (Hurme et al. 2007). Since patch occupancy may be affected by distances to neighbouring patches and their occupancy states, we also measured Euclidean (edgeto-edge) distance from each habitat patch: to the nearest patch without information on its occupancy status $(\mathrm{Np} ; \mathrm{m})$, and to the nearest occupied patch (Nop; m). All landscape metrics were calculated using algorithms developed for these analyses but based on spatial analysis tools in ArcInfo 8 (McCoy and Johnston 2001).

\section{Statistical analyses}

We analysed the patterns characterizing the occurrence of the flying squirrel by comparing variable values between occupied and unoccupied habitat patches in different years using descriptive statistics. We also modelled the occupancy of the flying squirrel in a habitat patch using logistic regression (Hosmer and Lemeshow 2000). For the logistic regression analysis, we selected four variables as the most important factors that could potentially influence flying squirrel occurrence, based on prior knowledge. These variables were: Patch size (ha) and Core-pro (\%), which represented patch quality (Hanski 1998, Hanski et al. 2000, Reunanen et al. 2002b), and Ph500 (\%) and Nop (m), which reflected quality of the surrounding matrix (Reunanen et al. 2002b, Hurme et al. 2005, 2007).

We used non-transformed variables and their main effects (no interactions) in modelling. Since independent variables were inter-correlated results must be interpreted with care. To reduce the likelihood of obtaining spurious results because of collinearity, we also avoided automated step-wise procedures. By contrast, we entered the variables into models using a biological hierarchy from patch scale variables to landscape scale variables. We first entered patch area in the model since large patches are more likely occupied than small patches because of chance only (see also van Apeldoorn et al. 1994). Second, we added quality of a patch, Core-pro, because it describes characteristics important for an individual flying squirrel and does not assume any landscape quality. Third, we added $\mathrm{Ph} 500$, since it describes the available habitat within a home range of an individual flying squirrel. Finally, we added distance to the nearest occupied patch, Nop, because the occupancy of a neighbouring habitat patch may affect the occupancy of a focal patch. Selection of the most parsimonious model was based on the Akaike's information criterion (AIC). AIC is the sum of deviance and two times the number of model parameters, whereby a smaller AIC value indicates the better model (Burnham and Andersson 2003).

Our purpose was to test the temporal consistency of a model with by examining if a model from an earlier year (2000) could be used to predict patch occupancy in 2004. Logistic regression is also an appropriate method for examining model transferability (Araújo and Guisan 2006). According to present knowledge, flying squirrels rarely live more than five years (Mäkelä 1996), so it was unlikely that the same individuals would inhabit the surveyed patches four years later. Predicted probabilities were divided to presence and absence based on a cut point of 0.5 . The model accuracy was estimated based on statistics recommended by Fielding and Bell (1997); including the area under the curve (AUC) of a threshold independent receiving operating characteristics (ROC) plot. In a ROC plot, sensitivity (true positive fraction) and 1-specificity (false positive fraction) are plotted and the AUC provides a measure of the model's overall accuracy. The AUC value ranges from 0.5 of no discrimination ability (random) to 1.0 of perfect discrimination ability (all cases predicted correctly). We also used Kappa K to estimate the overall agreement of the model with actual data. The Kappa $\mathrm{K}$ value ranges from 0.0 (no agreement) to 1.0 (excellent agreement). In addition, rates of false positive (predicted occupied but observed unoccupied) and false negative (predicted unoccupied but observed occupied) predictions were calculated.

The habitat patches surveyed during the years 20002004 were divided into three classes: continuously occupied, continuously unoccupied patches and patches in which occupancy state changed at least once during the study (variable-occupancy patches hereafter). Comparisons between these three classes were carried out using the nonparametric Kruskal-Wallis test. The variable-occupancy patches were surveyed a fourth time in 2006 for flying squirrel occurrence. Data from the variable-occupancy patches during 2000-2006 were used to examine temporal colonisation and abandonment events (see also van Apeldoorn et al. 1994).

The effect of timber harvesting on changes in patch occupancy states during 2000-2004 was examined by measuring the reduction in the amount of potential habitat within a 500-m buffer of each surveyed patch. The reduction in habitat (ha) was simplified into a dichotomous classification indicating whether habitat loss had (Yes), or had not (No) occurred. We used cross-tabulation (Yes-No) and classified pooled occupancy data in two ways. First, the data category was based on the same three occupancy status classes (continuously occupied, continuously unoccupied and variable-occupancy patches), and second, the classification scheme was based on colonization-abandonment events (colonization 0-1, abandonment 1-0, occupied 1-1 and unoccupied 0-0; between 2000 and 2004). The effects of forest clearing in the surrounding matrix on patch occupancy were analysed using a chi-square test with a Monte Carlo simulation (10000 simulated samples and a 
95\% confidence interval). Statistical analyses were conducted using SPSS (15.0).

\section{Results}

The potential habitat (total coverage of habitat patches) was reduced by $5.5 \mathrm{~km}^{2}(1.4 \%$ ) from 2000 to 2004 (Table 1). Four patches were completely clear cut between 2000 and 2002, and 11 patches between 2002 and 2004. Four of the cleared patches were occupied once (in 2000 or 2002) before being cut. One occupied patch was also divided into two separate patches by forest clearing (2002-2004). Of the 119 habitat patches existing in 2004, 18 were partially cleared, reducing their size by an average of $21.3 \%$ (average reduction in patch size $=23.3 \mathrm{ha}$, range $=0.5-135.1 \mathrm{ha}$ ).

The proportion of habitat patches occupied by flying squirrels was $36.1 \%$ (48 of 133) in 2000, 36.7\% (47 of 128 ) in 2002 and $52.1 \%$ (62 of 119) in 2004. Across the years, occupied patches were consistently of better quality, defined by larger sizes, total area of core habitat, higher volumes of deciduous trees, shorter distances to neighbouring patches, and higher percentage of potential habitat in the surrounding matrix (Supplementary material, Appendix 1). Patch size and distance to the nearest occupied patch (Nop) were consistently selected as significant predictors of patch occupancy in logistic regression models developed for each of the four survey years (Table 2), although some variation among models existed. The year 2002 differed from other years in that the proportion of core pixels (Corepro) and amount of potential habitat in the surrounding matrix $(\mathrm{Ph} 500)$ were also identified as significant predictors of patch occupancy.

Temporal generality of the logistic regression model using data from 2000 (with the variables Patch size and Nop [constant $=-0.954$, Patch size $\mathrm{B}=0.035$ and Nop $\mathrm{B}=-0.001]$ ), was evaluated based on the accuracy with which it predicted patch occupancies in 2004. The model for the year 2000 was moderately accurate predicting $77.4 \%$ of patch occupancies for the year 2000 (presence 50.0\%; absence $92.9 \%$; AUC $=0.83$ and $\mathrm{K} 0.47$ ), and its predictive accuracy for the test data in 2004 was $69.8 \%$ (presence $46.8 \%$, absence $94.7 \%$ ) with an AUC value of 0.81 and Kappa K 0.41. Rates of false negatives and false positives were also approximately equal in 2000 (50 and 7\%, respectively) and 2004 (53 and 5\%, respectively).

Results of the three surveys (2000-2004) showed that 36 patches were continuously occupied, 51 continuously unoccupied, and occupancy was variable in 32 (Fig. 1). Based on measured variables, the continuously occupied patches were of the best quality and continuously unoccupied patches of the lowest while the quality of variableoccupancy patches was intermediate (Table 3). However, unequivocal separation of variable-occupancy patches from the continuously unoccupied patches with measured variables was not possible. If the habitat suitability is determined by the observed occupancy of a patch in any year (continuously occupied and variable-occupancy patches), $57 \%$ of mature spruce-dominated forest patches in our study area were suitable for the flying squirrel. Numbers of continuously occupied and variable-occupancy patches were approximately equal, so changes in the occupancy of some habitat patches can be considered a typical feature of flying squirrel population dynamics in this landscape.

The 32 variable-occupancy patches were surveyed for the fourth time in 2006. Of these, 11 were occupied three times, 16 were occupied twice and 5 were occupied only once (Table 4). The percentage of flying squirrel occupancy within variable-occupancy patches varied: it was $34 \%$ in $2000,25 \%$ in $2002,84 \%$ in 2004 , and $75 \%$ in 2006 . There were eight colonisation and nine abandonment events between 2000 and 2002, twenty and three between 2002 and 2004, and three and four between 2004 and 2006. In total, there were about twice as many colonisation events (31) than abandonment events (16). The most common pattern (12 patches) was two unoccupied years followed by two occupied years (0-0-1-1) from 2000 to 2006.

The majority of forest clearings were carried out between 2002 and 2004, but nevertheless, the highest proportion of flying squirrel occupancy (52.1\%) was observed in 2004. In 2000-2004, there were significantly more clearings in the vicinity surrounding continuously occupied patches (within $500 \mathrm{~m}$ ), versus continuously unoccupied patches than would be expected by chance $\left(\chi^{2}=15.833, \mathrm{p}<0.001\right)$. However, the frequency of clearings around variableoccupancy patches did not deviate from the expected. Similarly, clearings were more common than expected around occupied (1-1) patches compared to unoccupied (0-0) patches (from 2000 to 2004), but were approximately equal to the expected rate around patches that were colonized (0-1) versus those where abandonment (1-0) occurred $\left(\chi^{2}=18.989, \mathrm{p}<0.001\right)$.

\section{Discussion}

Temporal changes in patch occupancy seemed to be a relatively typical pattern in our study landscape. During 2000-2004, there were 36 continuously occupied and 32

Table 1. Landscape metrics describing the study area during each of the three years that occupancy surveys were conducted (proportion, \%, of the total study area, $374.6 \mathrm{~km}^{2}$, and standard deviation of average, $\mathrm{SD}$, in parenthesis).

\begin{tabular}{lcrr}
\hline & 2000 & 2002 & 2004 \\
\hline Potential habitat $\left(\mathrm{km}^{2}\right)$ & $64.2(17.1 \%)$ & $61.2(16.3 \%)$ & $58.7(15.7 \%)$ \\
Matrix $\left(\mathrm{km}^{2}\right)$ & $310.4(82.9 \%)$ & $313.5(83.7 \%)$ & $316.0(84.3 \%)$ \\
Number of habitat patches in the area & 133 & 128 & 119 \\
Average habitat patch size $($ ha) & $58.1(175.2)$ & $57.9(174.2)$ & $60.2(177.9)$ \\
Average distance to a nearest patch $(\mathrm{m})$ & $178.5(210.4)$ & $185.4(218.8)$ & $224.2(287.6)$ \\
Average distance to a nearest occupied patch $(\mathrm{m})$ & $559.6(664.7)$ & $636.2(772.5)$ & $452.2(493.9)$ \\
\hline
\end{tabular}


Table 2. Results of logistic regression analyses for selected variables for each year survey data were collected.

\begin{tabular}{|c|c|c|c|c|c|c|}
\hline \multirow[t]{2}{*}{ Variables in a model ${ }^{\mathrm{a}}$} & \multicolumn{2}{|l|}{2000} & \multicolumn{2}{|l|}{2002} & \multicolumn{2}{|l|}{2004} \\
\hline & Accuracy $\%^{\mathrm{b}}(0 / 1)$ & $\mathrm{AIC}^{\mathrm{C}}$ & Accuracy \% (0/1) & $\mathrm{AIC}$ & Accuracy $\%(0 / 1)$ & $\mathrm{AIC}$ \\
\hline $\mathrm{Pa}$ & $76.7(94.1 / 45.8)$ & 136.4 & $78.1(93.8 / 51.1)$ & 126.1 & $68.9(82.5 / 56.5)$ & 136.4 \\
\hline $\mathrm{Pa}+$ Core-pro & $76.7(94.1 / 45.8)$ & 137.0 & 79.7 (92.6/57.4) & 120.5 & $69.7(80.7 / 59.7)$ & 136.8 \\
\hline $\mathrm{Pa}+\mathrm{Ph} 500$ & $75.9(92.9 / 45.8)$ & 136.1 & $78.1(93.8 / 51.1)$ & 125.3 & $68.1(78.9 / 58.1)$ & 137.2 \\
\hline $\mathrm{Pa}+\mathrm{Nop}$ & $77.4(92.9 / 50.0)$ & 129.3 & $77.3(90.1 / 55.3)$ & 116.5 & $73.1(78.9 / 67.7)$ & 126.2 \\
\hline $\mathrm{Pa}+$ Core-pro+Ph500 & $75.9(92.9 / 45.8)$ & 137.0 & $78.9(91.4 / 57.4)$ & 120.0 & $69.7(75.4 / 64.5)$ & 137.7 \\
\hline $\mathrm{Pa}+$ Core-pro $+\mathrm{Nop}$ & $76.7(91.8 / 50.0)$ & 130.3 & $78.9(88.9 / 61.7)$ & 112.7 & 74.8 (78.9/71.0) & 127.1 \\
\hline $\mathrm{Pa}+$ Core-pro $+\mathrm{Ph} 500+\mathrm{Nop}$ & $76.7(91.8 / 50.0)$ & 132.3 & $78.9(88.9 / 61.7)$ & 114.6 & $74.8(75.4 / 71.0)$ & 128.2 \\
\hline
\end{tabular}

${ }^{\mathrm{a}} \mathrm{Pa}=$ patch area (ha), Core-pro $=$ proportion of core habitat in a patch $(\%), \mathrm{Ph} 500=$ area of potential habitat within $500 \mathrm{~m}$ radius around a patch (ha), Nop $=$ distance to the nearest occupied patch $(\mathrm{m})$.

${ }^{b}$ Accuracy shows the correctly predicted overall occupancy of habitat patches (percentage of correctly predicted unoccupied/occupied patches in parenthesis) by the variables included in the model.

${ }^{\mathrm{C}} \mathrm{AIC}=\mathrm{Akaike}$ 's information criterion (sum of deviance and two times the number of variables in a model). The model having the lowest AIC (each year) is shown in bold.

variable-occupancy patches, while 51 patches were continuously unoccupied. Thus, more than half of the mature spruce-dominated forest patches in our study area could be regarded as suitable for the flying squirrel, being occupied at least once. This is more than the approximately 35\% occupancy often observed in northern Finland in single year surveys (Reunanen et al. 2002b, Hurme et al. 2005). A dynamic patch occupancy pattern is thus an important aspect in population dynamics, and points out the importance of long-term surveys to detect the actual patch network.

In all study years, occupied patches were consistently of better quality in terms of larger size, higher volume of deciduous trees, and shorter distances to other occupied patches (see also Hurme et al. 2007). The temporal perspective revealed that continuously occupied patches were generally among those of the highest quality, and continuously unoccupied patches were among the lowest, while patches with variable occupancy tended to be intermediate patches based on the measured variables. We assume that flying squirrels in our study area constitute one local population and that colonisations and abandonments of the patches reflect movements and deaths of individual squirrels. It is still possible that patches of high quality function as source patches that support patches of lower habitat quality (sensu Pulliam 1988). Large, continuously occupied patches are likely to be fundamental source habitats that maintain the local patch network while small and low quality patches, as well as patches that were occupied every year, may remain occupied due to regular immigration. Selonen et al. (2007) found that dispersing flying squirrels tended to settle on patches lower in quality than the ones in which they were born. The sink patches apparently have an important role for the local population dynamics (Pulliam 1988, Foppen et al. 2000, Ozgul et al. 2006) and are thus essential parts of the habitat network.

At times, suitable habitat patches seem to be unoccupied. In territorial beavers, for example, only $20 \%$ of their colonies were consistently occupied during a study period of $11 \mathrm{yr}$ (Fryxell 2001). In northern spotted owls variation in site occupancy may affect occupancy modeling results (Olson et al. 2005), while in sea birds, island abandonment rates may vary greatly from year to year (Martínez-Abraín et al. 2003). Haila et al. (1993) pointed out that for birds local turnover may reflect a change in territory location from year to year and thus, may be disconnected from population dynamics. Flying squirrels, especially the breeding females, have been found to be site-tenacious (Hanski 1998, Hanski et al. 2000) and therefore, the observed turnover in our study area probably was not due to territorial relocation.

However, repeated field surveys were required to identify patches with variable occupancy. The number of colonisation and abandonment events varied from one to two times without any clear pattern in timing (see also Table 4), but we could not relate these changes in occupancy to the variables we used. Better data on habitat attributes that indicate habitat quality can help develop modelling

Table 3. Comparison of the variables from the year 2004 data using averages (and 95\% confidence interval) based on the occurrence of the Siberian flying squirrel during the three years of the study. Kruskall-Wallis test statistics $(H)$ and its significance $(p)$ are shown $(D F=2)$.

\begin{tabular}{lccrr}
\hline & $\begin{array}{c}\text { Continuously occupied } \\
(\mathrm{n}=36)\end{array}$ & $\begin{array}{c}\text { Variable-occupancy } \\
(\mathrm{n}=32)\end{array}$ & $\begin{array}{c}\text { Continuously unoccupied } \\
(\mathrm{n}=51)\end{array}$ & $\mathrm{H}(\mathrm{p})$ \\
\hline Patch size (ha) & $162.5(60.6-264.5)$ & $20.4(14.8-26.0)$ & $12.9(9.7-16.2)$ & $28.636(0.000)$ \\
Core-area (ha) & $34.7(11.9-57.5)$ & $3.0(2.0-3.9)$ & $1.7(1.2-2.3)$ & $31.075(0.000)$ \\
Core-pro (\%) & $17.3(15.3-19.2)$ & $13.6(12.2-15.0)$ & $11.6(10.6-12.7)$ & $23.712(0.000)$ \\
Spruce $\left(\mathrm{m}^{3} \mathrm{ha}^{-1}\right)$ & $53.7(50.0-57.3)$ & $46.2(42.4-49.9)$ & $42.2(39.2-45.2)$ & $21.022(0.000)$ \\
Deciduous $\left(\mathrm{m}^{3} \mathrm{ha}^{-1}\right)$ & $13.2(12.1-14.3)$ & $11.5(10.4-12.6)$ & $10.5(9.7-11.3)$ & $13.043(0.001)$ \\
Np (m) & $170.1(68.4-271.8)$ & $232.4(139.0-315.8)$ & $257.1(174.1-340.2)$ & $5.140(0.077)$ \\
Nop (m) & $201.3(97.5-305.1)$ & $385.8(218.8-552.9)$ & $670.9(522.1-819.7)$ & $25.124(0.000)$ \\
Ph500 (\%) & $26.2(22.9-29.6)$ & $17.0(13.6-20.4)$ & $15.2(12.6-17.8)$ & $26.414(0.000)$ \\
Matrix500 (\%) & $73.8(70.4-77.1)$ & $83.0(79.6-86.4)$ & $84.8(82.2-87.4)$ & $26.414(0.000)$ \\
\hline
\end{tabular}




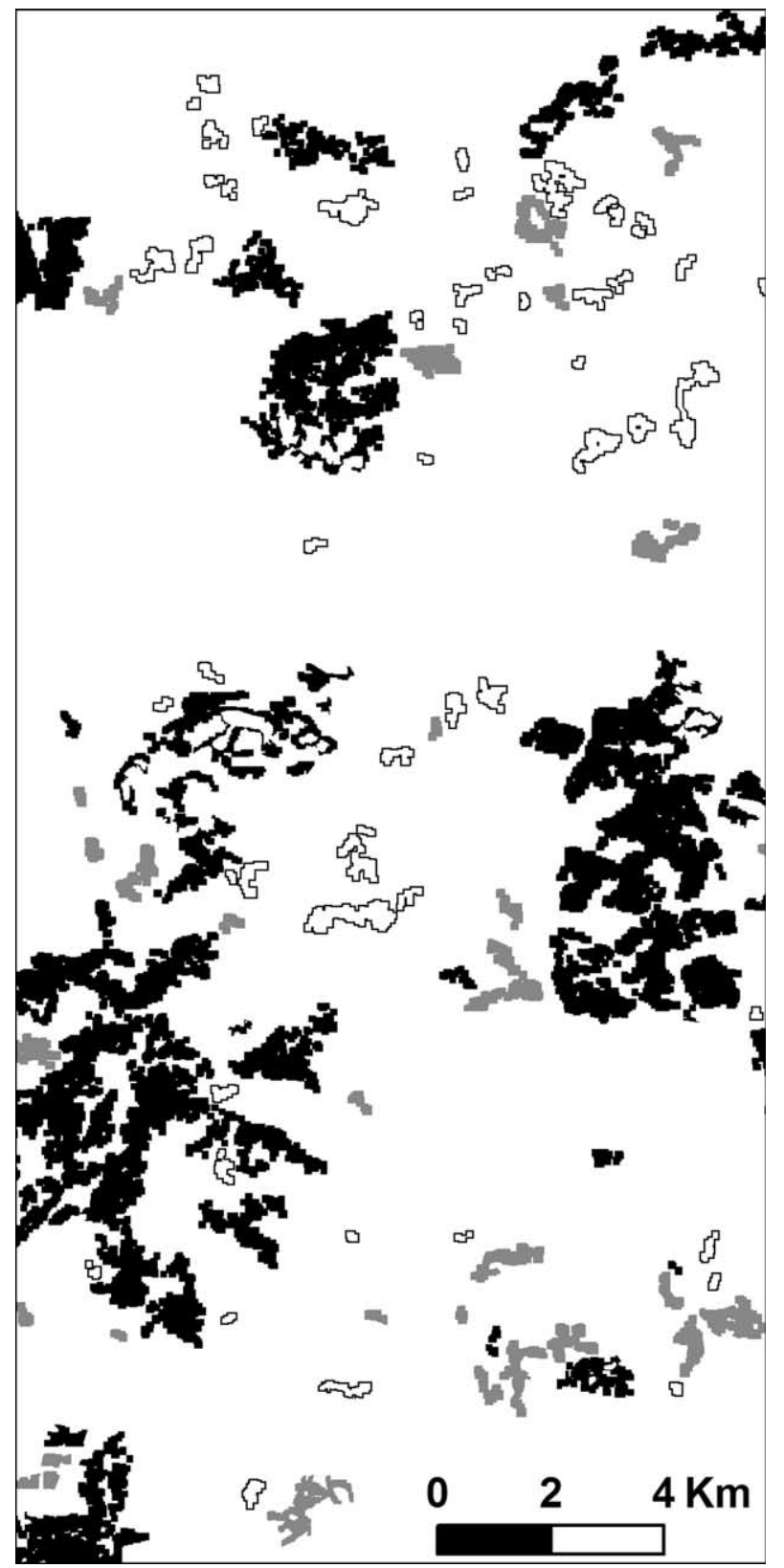

Figure 1 . The study area showing the potential habitat patches in $2004(\mathrm{n}=119)$ and their occupancy pattern: continuously occupied (black), continuously unoccupied (white) and variableoccupancy (gray) during 2000-2004.

approaches. Inclusion of data on aspen, the potential cavity and nesting trees for the flying squirrel, might better identify the variable-occupancy patches, potentially eliminating the need for repeated surveys. There were often more aspens within habitat patches rich in deciduous trees in the study area (unpubl.; see also Reunanen et al. 2002a), but information on aspen was not distinguished reliably enough from other deciduous trees from satellite images or MS-NFI data.

Patch size and distance to the nearest occupied patch were the main factors explaining patch occupancy each year. In the red squirrel Sciurus vulgaris the probability of the patch being occupied was affected by size and distance to other occupied patches (van Apeldoorn et al. 1994 but see
Table 4. Observed sequences of occupancies $(1=$ occupied, $0=$ unoccupied) in patches with occupancy that varied over the study period $(\mathrm{n}=32)$.

\begin{tabular}{lcccc}
\hline 2000 & 2002 & 2004 & 2006 & $\begin{array}{c}\text { Patches having this } \\
\text { sequence }\end{array}$ \\
\hline 0 & 1 & 1 & 1 & 5 \\
1 & 0 & 1 & 1 & 5 \\
1 & 1 & 0 & 1 & 1 \\
0 & 0 & 1 & 1 & 12 \\
1 & 0 & 1 & 0 & 2 \\
1 & 0 & 0 & 1 & 1 \\
0 & 1 & 1 & 0 & 1 \\
1 & 0 & 0 & 0 & 2 \\
0 & 0 & 1 & 0 & 1 \\
0 & 1 & 0 & 0 & 2 \\
\hline
\end{tabular}

Verbeylen et al. 2003). The model for 2002 included the patch quality variable (Core-pro), unlike the models for 2000 and 2004. Although overall percent of occupancy did not vary between 2000 and 2002, the proportion of variable-occupancy patches that were occupied in 2002 was the lowest $(25 \%)$. This finding may suggest that overall abundance was lower in 2002 because low quality patches are likely to become empty first when the population density declines. van Apeldoorn et al. (1994) found that in years of presumed low density, occupancy of the red squirrel was more strongly linked to habitat quality than in other years (but see Verbeylen et al. 2003). Thus, it is possible that the effect of patch quality on occupancy may be detectable only when the population density is low (Gaston et al. 2000).

We found that a logistic regression model from 2000 was also rather accurate in predicting occupancy in the year 2004. The similar accuracy in both years suggests that our model can be temporally generalised and that single-year surveys can point out some of the most important features explaining the flying squirrel occupancy. On the other hand, false negative prediction rates were relatively high with regard to both training and test data. This was partially due to the occupancy dynamics in intermediate quality patches where occupancy may be influenced more by population demography than habitat characteristics in a patch. Therefore, the risk of failing to identify all potentially suitable patches in the landscape using a single-year survey model is considerable. Uncertainties can even increase if the model is generalised to other geographical regions (but see Menéndez and Thomas 2006).

The proportion of occupied patches was relatively high every year (36-52\%) in our study area, and it tended to increase in time (more colonisations than abandonments), particularly between 2002 and 2004. These findings suggest some changes in the local population density, and may indirectly reflect an increase in abundance. Martínez-Abraín et al. (2003), for example, found that island abandonment of Audouin's gulls was much lower when the colony was increasing than when it was declining. The dominance of colonisations in habitat patches also suggests that movement between habitat patches is regular and hence, the study landscape can be considered functionally connected (see also Hurme et al. 2007). However, our data cannot be used to distinguish a population increase from fluctuations in population density. This is because density may increase 
without an increase in population size if, for example, individuals move to remaining patches after habitat loss (Lovejoy et al. 1986).

There were more clear cuttings in the vicinity of patches that were continuously occupied (see also Hurme et al. 2007), but we found no evidence that harvesting affected patch occupancy. It is possible that present population dynamics in our study area depend more on abiotic effects (weather) or biotic interactions (predators, parasites, diseases, etc.) than on changes in landscape structure. The lack of association between patch occupancy and landscape change may also suggest that there is adequate habitat area available in our study landscape. Alternatively, the population may be in disequilibrium because of recent cuttings, and it is possible that our follow-up period has been too short to detect the effects of changes in landscape structure.

The habitat threshold denotes minimum area requirements that support long-term persistence of a species in the landscape (Andrén 1994, Reunanen et al. 2004). Habitat threshold assessment is based on observed or expected species-habitat associations. However, determination of habitat thresholds is problematic because a threshold depends much on what is actually considered as habitat and how that habitat is assigned as occupied. In our landscape, no patches above $300 \mathrm{~m}$ a.s.l. were observed occupied in an earlier study (Hurme et al. 2007) or during this follow-up study (data not shown). Also during 20002004, there were almost as many temporarily occupied patches as continuously occupied ones. If we consider only spruce-dominated habitat situated below $300 \mathrm{~m}$ a.s.l., continuously occupied patches covered $8.5 \%$ and variableoccupancy patches $1.4 \%$ (500 ha) of the total landscape. Hence, in a single-year survey, up to 500 ha of potentially suitable habitat could have gone undetected and led to underestimation of habitat availability. Estimation of available habitat is therefore affected by changes in the occupancy pattern, which should be carefully considered in landscape planning for species conservation.

In this study, repeated surveys revealed considerable variation in the habitat occupancy status of the flying squirrel, and improved our understanding of patch occupancy dynamics and patch quality for the focal species. Habitat patch and landscape characteristics that explain the occurrence of the flying squirrel were relatively consistent among years, and a statistical model using patch occupancy data from a single-year survey was relatively robust for predicting species occurrence after a four year time-lag. However, due to the dynamic patch-occupancy pattern, the model failed to consistently identify variable-occupancy patches that repeated surveys revealed over the course of the study period. This challenges landscape management planning because a single survey does not necessarily detect seldom used habitat patches that may be important for the local population. When planning the conservation of functional habitat networks at broader scales, habitat patches that are presently empty should also be included in a network (see also Martínez-Abraín et al. 2003). A better understanding of patterns and processes of the flying squirrel populations in Finland still requires follow-up studies in different geographical regions.
Acknowledgements - We thank the two referees for their helpful comments and M. Mönkkönen for checking the language. E. Tomppo and colleagues in the Forest Research Inst. of Finland are acknowledged for allowing the use of MS-NFI data, and Metsähallitus for giving the forest regeneration data for updating our landscape. We warmly thank R. Gasol Escuer, M. Ukkola, J. Seppänen, J. Sitte and D. Gabrikova for their assistance in the field. Financial support was provided by the Univ. of Oulu, the Academy of Finland (project no 52772), the Ministry of Agriculture and Forestry of Finland (project no 25058) and the EnviroNet, joint graduate school of the Univ. of Oulu and NorNet (to E.H.), and by the Finnish Biodiversity Research Programme FIBRE (to P.R.).

\section{References}

Ahti, T. et al. 1968. Vegetation zones and their sections in northwestern Europe. - Ann. Bot. Fenn. 5: 169-211.

Andrén, H. 1994. Effects of habitat fragmentation on birds and mammals in landscapes with different proportion of suitable habitat: a review. - Oikos 71: 340-346.

Araújo, M. B. and Guisan, A. 2006. Five (or so) challenges for species distribution modeling. - J. Biogeogr. 33: 1677-1688.

Burnham, K. P. and Andersson, D. R. 2003. Model selection and inference: a practical information-theoretic approach. - Springer.

Fielding, A. H. and Bell, J. F. 1997. A review of methods for assessment of prediction errors in conservation presence/ absence models. - Environ. Conserv. 24: 38-49.

Foppen, R. P. B. et al. 2000. Understanding the role of sink patches in source-sink metapopulations: reed warbler in an agricultural landscape. - Conserv. Biol. 14: 1881-1892.

Fryxell, J. M. 2001. Habitat suitability and source-sink dynamics of beavers. - J. Anim. Ecol. 70: 310-316.

Gaston, K. J. et al. 2000. Abuncande-occupancy relationships. - J. Appl. Ecol. 37: 39-59.

Guisan, A. and Zimmermann, N. E. 2000. Predictive habitat distribution models in ecology. - Ecol. Model. 135: 147-186.

Guisan, A. and Thuiller, W. 2005. Predicting species distribution: offering more than simple habitat models. - Ecol. Lett. 8: 993-1009.

Haila, Y. et al. 1993. Turnover of breeding birds in small forest fragments: the "sampling" colonization hypothesis corroborated. - Ecology 74: 714-725.

Hanski, I. K. 1998. Home range and habitat use in the declining flying squirrel Pteromys volans in managed forests. - Wildl. Biol. 4: 33-46.

Hanski, I. K. et al. 2000. Home range size, movements, and nestsite use in the Siberian flying squirrel (Pteromys volans). - J. Mammal. 81: 798-809.

Hosmer, D. W. and Lemeshow, S. 2000. Applied logistic regression, 2nd ed. - Wiley.

Hurme, E. et al. 2005. Building and evaluating predictive occupancy models for the Siberian flying squirrel using forest planning data. - For. Ecol. Manage. 216: 241-256.

Hurme, E. et al. 2007. Local habitat patch pattern of the Siberian flying squirrel in a managed boreal forest landscape. - Ecography 30: 277-287.

Joseph, L. N. et al. 2006. Presence-absence versus abundance data for monitoring threatened species. - Conserv. Biol 20: 16791687.

Lovejoy, T. E. et al. 1986. Edge and other effects of isolation on Amazon forest fragments. - In: Soulé, M. E. (ed.), Conservation biology: the science of scarcity and diversity. Sinauer. pp. 257-285.

Mäkelä, A. 1996. Liito-oravan (Pteromys volans L.) lisääntymisbiologiasta. (About the breeding biology of the Siberian 
flying squirrel.) - Maailman Luonnon Säätiön WWF Suomen Rahaston Raportteja 8: 63-65, in Finnish.

Martínez-Abraín, A. et al. 2003. Modeling temporal and spatial colony-site dynamics in a long-lived seabird. - Popul. Ecol. 45: 133-139.

McCoy, J. and Johnston, K. 2001. Using $\operatorname{ArcGIS}^{\mathrm{TM}}$ spatial analyst. - ESRI, USA.

Menéndez, R. and Thomas, C. D. 2006. Can occupancy patterns be used to predict distributions in widely separated geographic regions? - Oecologia 149: 396-405.

Mönkkönen, M. et al. 1997. Landscape characteristics associated with the occurrence of the flying squirrel Pteromys volans in old-growth forests of northern Finland. - Ecography 20: 634642.

Nielsen, S. E. et al. 2005. Can models of presence-absence be used to scale abundances? Two case studies considering extremes in life history. - Ecography 28: 197-208.

Olson, R. G. et al. 2005. Modeling of site occupancy dynamics for northern spotted owls, with emphasis on the effects of barren owls. - J. Wildl. Manage. 69: 918-932.

Ozgul, A. et al. 2006. Effects of patch quality and network structure on patch occupancy dynamics of a yellow-bellied marmot metapopulation. - J. Anim. Ecol. 75: 191-202.

Pollock, J. F. 2006. Detecting population declines over large areas with presence-absence, time-to-encounter, and count survey methods. - Conserv. Biol. 20: 882-892.

Pulliam, H. R. 1988. Sources, sinks, and population regulation. - Am. Nat. 132: 652-661.

Randin, C. F. et al. 2006. Are niche-based species distribution models transferable in space? - J. Biogeogr. 33: 1689-1703.

Rassi, P. et al. (eds) 2001. The red list of Finnish species. Ministry of the Environment, and Finnish Environment Inst., Finland, in Finnish with English summary.

Reunanen, P. et al. 2000. Managing boreal forest landscapes for the flying squirrel. - Conserv. Biol. 14: 218-226.

Download the Supplementary material as file E5373 from $<$ www.oikos.ekol.lu.se/appendix $>$.
Reunanen, P. et al. 2002a. Habitat requirements of the Siberian flying squirrel in northern Finland: comparing field survey and remote sensing data. - Ann. Zool. Fenn. 39: 7-20.

Reunanen, P. et al. 2002b. Predicting occupancy for the Siberian flying squirrel in old-growth forests. - Ecol. Appl. 12: 11881198.

Reunanen, P. et al. 2004. Assessing landscape thresholds for the Siberian flying squirrel. - Ecol. Bull. 51: 277-286.

Scott, J. M. et al. (eds) 2002. Predicting species occurrences. Issues of accuracy and scale. - Island Press.

Selonen, V. and Hanski, I. K. 2004. Young flying squirrels (Pteromys volans) dispersing in fragmented forests. - Behav. Ecol. 15: 564-571.

Selonen, V. and Hanski, I. K. 2006. Habitat exploration and use in dispersing juvenile flying squirrels. - J. Anim. Ecol. 75: 1440-1449.

Selonen, V. et al. 2001. Space use of the Siberian flying squirrel Pteromys volans in fragmented forests. - Ecography 24: 588600.

Selonen, V. et al. 2007. Natal habitat-biased dispersal in the Siberian flying squirrel. - Proc. R. Soc. B 274: 2063-2068.

Tomppo, E. 1993. Multi-source National Forest Inventory of Finland. - The Finnish Forest Research Inst., Research Papers 444: 52-59.

Tosh, C. A. et al. 2004. Estimating the abundances of large herbivores in the Kruger National Park using presence-absence data. - Anim. Conserv. 7: 55-61.

van Apeldoorn, R. C. et al. 1994. Distribution and dynamics of the red squirrel (Sciurus vulgaris L.) in a landscape with fragmented habitat. - Landscape Ecol. 9: 227-235.

Verbeylen, G. et al. 2003. Patch occupancy, population density and dynamics in a fragmented red squirrel Sciurus vulgaris population. - Ecography 26: 118-128. 